\title{
Research on Evaluation Method of Distribution Network State
}

\author{
Liu Shiqi ${ }^{1}$, Yang Yaojia ${ }^{1}$, Peng Hui ${ }^{1, a,{ }^{*}}$ \\ ${ }^{1}$ Scool of Electrical Engineering Wuhan University, Wuhan, P.R. China, \\ ahpeng@whu.edu.cn
}

\begin{abstract}
Keywords: fuzzy comprehensive evaluation, evaluation matrix, single-value processing,
\end{abstract} distribution network

\begin{abstract}
In recent years, power grid assessments are more and more important with the continuous development of distribution network. Although much research has been done about evaluation method of medium voltage distribution network, it is hard to establish a united evaluation system of distribution network and the corresponding evaluation algorithm. Fuzzy comprehensive evaluation method has been used in this paper to assess the distribution network. Establishment of comprehensive evaluation matrix and fuzzy comprehensive evaluation can be done on the basis of established factors sets, evaluation sets, and weight sets. The evaluation score of each partition is obtained by single-value processing the result vector, as well as the analysis of regional distribution network running state rank and the influence factors in the region running state. Finally, the feasibility of this process is validated by assessing a regional distribution network.
\end{abstract}

\section{Introduction}

At present, the commonly used evaluation methods include expert analysis, analytic hierarchy process, ideal point method, support vector machine multi classifier, Bias classifier method, utility criterion method, risk assessment method and so on. These methods are both the combination of quantitative and qualitative. The expert analysis method is widely accepted because it' s simple and easy to explain. But with strong subjectivity, and it can not make the results more scientific and objectively reflect the characteristics of the system.

The traditional evaluation method has the problem of weight determination. On the one hand, most of these evaluation methods are based on the experience of the experts, with a greater subjective randomness. On the other hand, these methods are very complex and difficult to solve in the calculation and weight calculation. The results that project evaluation obtained are becoming more and more significant with the development and general application of neural network technology. Still, there are several shortcomings when applying the neural network technology: firstly, it is difficult to determine the network structure. What' $s$ more, the model generalization is poor. And the quantity and quality of the sample have certain requirements.

According to the statistical results of the widely used evaluation method recently, support vector machine multi classifier method and the Bias classification method is more common. Several common evaluation methods will be introduced in the following paper.

\section{Selection of evaluation method}

In this section, several common evaluation methods will be introduced as well as comparison with each other, and a suitable evaluation method for the distribution network will be chose then.

Support vector machine method. Support vector machine (Vector Machine Support) [1,2], which was proposed by Vapnik in 1995, is a machine learning method based on statistical learning theory that has relatively good performance indicators. Generally speaking, the support vector machine (SVM) projects the original space to a high dimensional space through the nonlinear transform of the inner product function, and solves the optimal classification face of the space (generalized). Support vector machine model is shown in figure 1.

SVM has an important theoretical basis and generalization performance for small sample data. 


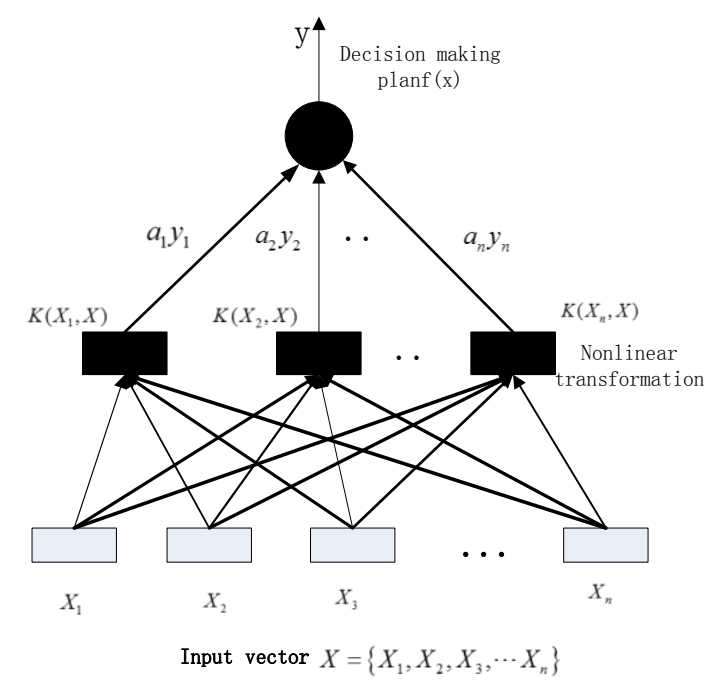

Fig. 1 Support vector machine

Bias network method. The Bias network $[3,4]$ is proposed by Pearl in 1986, which is a simple and effective new uncertain knowledge representation model based on probability calculation. Bias (Network Bayesian), also known as Networks Belief (Networks Probabilistic), is an extension of the Bayes method, which provides a natural way to express the causal relationship, and it can be used as a graphical model representing multi variable joint probability and as well as to find the potential relationship between the data.

In practical engineering, there are many factors that are not deterministic. These factors have two aspects: one is randomness, the two is fuzziness [5]. For this kind of things with uncertainty and ambiguity, a special theory method is supposed to be applied, that is the fuzzy mathematics method.

Fuzzy evaluation method. In order to solve the uncertainty problems of complex systems, an effective tool---fuzzy set theory is proposed by L.A.Zadch [6-7].

According to the evaluation index of the evaluation object, the steps of the fuzzy comprehensive evaluation method for the comprehensive evaluation of the state level are shown as following:

1) Determine the object of the evaluation $X$;

2) Set up factor set $U$. It is supposed that the number of effect factor is $n$, (namely evaluation index system), which are $u_{1}, u_{2}, \ldots, u_{n}$ respectively, then the factor set is recorded as $U=\left\{u_{1}, u_{2}, \ldots, u_{n}\right\}$, and the factor vector corresponded with factor set is recorded as $U=\left\{u_{1}, u_{2}, \ldots, u_{n}\right\}$;

3) Set up evaluation set $V$ and set $v_{i}$ as the evaluation grade and set $m$ as the level number. The evaluation set is recorded as $V=\left\{v_{1}, v_{2}, \ldots, v_{m}\right\}$, which is used to indicate the level of the evaluation;

4) Set up weight set $W$. Weight is a discription about the relatively important degree between one factor in factor set $U$ and the evaluation object. Let the weights of $u_{1}, u_{2}, \ldots, u_{n}$ are $w_{1}, w_{2}, \ldots, w_{n}$ and meet the following relationship: $\sum_{i=1}^{n} w_{i}=1$. The weight set is recorded as $W=\left\{w_{1}, w_{2}, \ldots, w_{n}\right\}$;

5) Set up comprehensive evaluation matrix $R$ that is the fuzzy relational matrix of factor set $U$ and evaluation set $V$. It is also called fuzzy membership matrix. It is defined that $R=\left(r_{i j}\right)_{n \times m}$ as well as $\sum_{j=1}^{m} r_{i j}=1$ by normalization. In this formula, $r_{i j}$ is the membership degree of the 
influence factors $u_{i}$ in the evaluation grade $v_{j}$. That is, $r_{i j}$ is a quantitative index the degree of $u_{i}$ belongs $v_{j}$.

6) Do fuzzy comprehensive evaluation calculation like this:

$$
\begin{aligned}
B & =W \circ R=\left(w_{1}, w_{2}, \ldots, w_{n}\right) \circ\left(r_{i j}\right)_{n \times m} \\
& =\left(b_{1}, b_{2}, \ldots, b_{m}\right)
\end{aligned}
$$

In this equation:

$b_{j}=\sum\left(w_{i} \circ r_{i j}\right) j, j=1,2, \ldots, m$

The “०” in $B=W \circ R$ means fuzzy operators, and using different operators will get different results. Here are some of the commonly used operators:

(1) "Main factor determining type" operator, denoted as $M(\wedge, \vee)$, so $b_{j}=\underset{i=1}{\vee}\left(w_{i} \wedge r_{i j}\right) j, j=1,2, \ldots, m$.

(2) "Principal factor prominent type" operator, denoted as $M(\circ, \vee)$, so $b_{j}=\stackrel{n}{\vee}\left(w_{i=1} \times r_{i j}\right) j, j=1,2, \ldots, m$.

(3) “Unbalanced average type" operator, denoted as $M(\wedge,+)$, so $b_{j}=\sum_{i=1}^{n}\left(w_{i} \wedge r_{i j}\right) j, j=1,2, \ldots, m$.

(4) "Weighted averaging type" operator, denoted as $M(\circ,+)$, so $b_{j}=\sum_{i=1}^{n}\left(w_{i} \times r_{i j}\right) j, j=1,2, \ldots, m$.

The fourth operator is adopted in this paper, because of the weighting function can take the weight of each index into account and will not give up any indicator that may affect the results.

7) Single valued comprehensive evaluation result vector

Finally, the fuzzy comprehensive evaluation result that we got is a fuzzy vector. It is supposed to be converted into a percentage of percentile. So the uniformization processing with comprehensive evaluation results of fuzzy vector is needed, that is we need to give each grade a score and then we find the weighted average of the results in fuzzy evaluation result $B$ and the corresponding grade score to calculate the final score.

Set

$$
B=\left(b_{1}, b_{2}, \ldots, b_{l}\right)
$$

Set $l$ level from high to low order and give a score of $c_{1}, c_{2}, \ldots, c_{l}$, they have the same D-value. So the comprehensive evaluation result vector through uniformization processing can be expressed as:

$$
c=\sum_{k=1}^{l} b_{k}^{t} c_{k}
$$

In this equation: $t=1$ or $t=2$, it is used to control the undetermined coefficient of the bigger $b_{k}$.

8) Make the corresponding decision according to the comprehensive evaluation results of the evaluation object .

\section{Steps of distribution network state evaluation}

According to the above sections, it can be summarized that the steps of the distribution network state evaluation includes the distribution network partition [8-10], the selection of the state variables, the establishment of the weight, the establishment of the running state level. And the detailed flow chart is shown in Figure 2. 


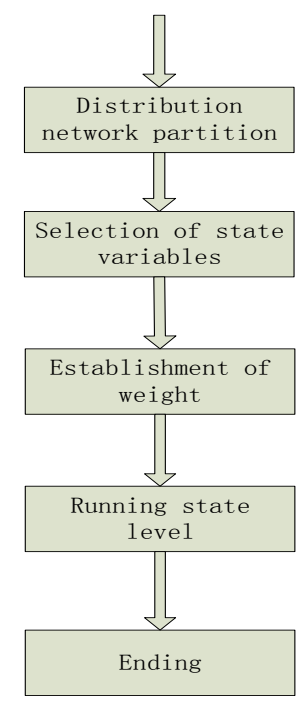

Fig. 2 Distribution network state evaluation process

\section{Distribution network partition}

The distribution network can be partitioned in accordance with the two levels of the way to partition. According to the geographical conditions and administrative system, the distribution network can be divided into several regions. The division of the second level is carried out based on the division of the first level. In this paper, the effect of each component failure in the same region is the same as that of this region and the other region and according to the two partition principle and the partition algorithm of the adjacency matrix, the method of distribution network partition is given.

Definition of adjacency matrix [11]:

$L_{i j}=\left\{\begin{array}{lr}0 & \text { No parent-child relationship between node } \mathrm{i}-\mathrm{j} \\ 1 & \text { There is an automatic switch between } \mathrm{i}-\mathrm{j} \\ -1 & \text { There is a manual switch between } \mathrm{i}-\mathrm{j} \\ 2 & \text { There is a connection between the branch } \mathrm{i}-\mathrm{j} \text { but no switch }\end{array}\right.$

The selection of state assessment of distribution network

Based on the evaluation of the status of the electrical equipment in the distribution network, it is time to study the evaluation method of the overall operating state of the distribution network. After taking into account the factors of the environment, weather conditions, operating modes, load changes, the operating state of the electric equipment in the distribution network and so on, we analyze the influence of these factors on the overall operating state of the distribution network and the extent of the impact. Then, 5 rational distribution network state variables are given: distribution network connection mode, load level, electrical equipment operation status, weather factors and other factors.

The establishment of state evaluation weight of distribution network

Extension analytic hierarchy process is used to divide the state evaluation index system of distribution network into two levels. According to the weight of the questionnaire [12], an important degree of interval is given by 6 experts or technical personnel which is obtained by comparison of any two of the indicators in each layer. Then the extension interval number judgement matrix is got.

$$
a_{i j}^{t}=\left(a_{i j}^{-t}, a_{i j}^{+t}\right),\left(i, j=1,2, . . n_{k} ; t=1,2, \ldots, T\right)
$$

Set the above equation to be the interval that the expert $t$ gives. According the following equation, $A^{t}=\frac{1}{T} \otimes\left(a_{i j}^{1}+a_{i j}^{2}+\ldots+a_{i j}^{T}\right)$, the $k^{\text {th }}$ layer comprehensive extension interval number 
judgement matrix is obtained, $A=<A^{-}, A^{+}>$. Then calculate the weight vector. It could be shown as follows:

$$
W^{k}=P^{k} P^{k-1} \cdots P^{3} W^{2}
$$

Establish the comprehensive evaluation matrix $R$. It is the fuzzy relation matrix of the factor set $U$ and the evaluation set $V$ and also named as fuzzy membership matrix. Carry out fuzzy comprehensive evaluation. That is:

$$
\mathrm{B}=\mathrm{W} \circ R=\left(w_{1}, w_{2}, \cdots, w_{n}\right) \circ\left(r_{i j}\right)_{n \times m}=\left(b_{1}, b_{2}, \cdots, b_{m}\right)
$$

In this formula:

$$
b_{j}=\sum\left(w_{i} \circ r_{i j}\right) j=1,2, \cdots, m
$$

In the fuzzy operator $\mathrm{B}=\mathrm{W} \circ R$, “०” adopts the weighted average operator $M(\circ,+)$. So, $b_{j}=\sum_{i=1}^{n}\left(w_{i} \times r_{i j}\right) j=1,2, \cdots, m$.

Finally, the fuzzy comprehensive evaluation result is a fuzzy vector[13]. It is supposed to be converted into a fraction of a percentage point. So the uniformization processing with comprehensive evaluation results of fuzzy vector is needed, that is we need to give each grade a score and then we find the weighted average of the results in fuzzy evaluation result $B$ and the corresponding grade score to calculate the final score.

Set

$$
B=\left(b_{1}, b_{2}, \ldots, b_{l}\right)
$$

Set $l$ level from high to low order and give a score of $c_{1}, c_{2}, \ldots, c_{l}$, they have the same $\mathrm{D}$-value. So the comprehensive evaluation result vector through uniformization processing can be expressed as :

$$
c=\sum_{k=1}^{l} b_{k}^{t} c_{k}
$$

In this equation: $t=1$ or $t=2$, it is used to control the undetermined coefficient of the bigger $b_{k}$.

\section{Case analysis}

In order to verify the partition algorithm and the evaluation method of the distribution network, in this section, the distribution network in a certain area is analyzed as an example.

Firstly, primary partition of the whole distribution network is done at beginning. The distribution network will be divided into several regions according to the geographical conditions. In this paper, the main consideration for division is electrical connection. Double power hand in hand and take account of the geographical conditions, then divide them into several first class regionals. Select one of the regions to carry out the secondary regional division and evaluation.

Secondly, secondary regional division and assessment will be done which selected from primary partition.

Take I region as an example to analyze concretely: the wiring way used in network is the power of the hand in hand. In August, the average load level is $73.5(M W)$, the weather level is normal, the geographical factors are the plain areas, the human factors have no accident.

The final evaluation score after the single value of the evaluation result of the fuzzy vector is obtained at last. We can analyze the operation status of the regional distribution network through the score of each partition, and get the weaker link in China. At the same time, we can find out the 
influence factors of regional evaluation scores on the region, and the analysis results can be used as the basis for decision makers.

The corresponding score and index status of each partition are shown in Table 1.

Table 1 The corresponding score and index status of each partition

\begin{tabular}{|c|c|c|}
\hline $\begin{array}{c}\text { partiti } \\
\text { on }\end{array}$ & $\begin{array}{c}\text { Averag load } \\
\text { (MW) }\end{array}$ & score \\
\hline 1 & 72.4 & 98.364 \\
\hline 2 & 125.3 & 98.154 \\
\hline 3 & 52.9 & 98.118 \\
\hline 4 & 27.8 & 97.726 \\
\hline 5 & 0 & 99.976 \\
\hline 6 & 0 & 99.976 \\
\hline 7 & 9.3 & 99.776 \\
\hline 8 & 0 & 99.976 \\
\hline 9 & 87.4 & 99.456 \\
\hline 10 & 74.4 & 99.354 \\
\hline 11 & 40.9 & 99.864 \\
\hline \multicolumn{2}{|c|}{ Footnote:Connection mode is hand in hand, } \\
\hline
\end{tabular}

It can be seen from the table that the score of the eleven partitions are all higher than 95 points, which are normal state, indicating that the current operation of the distribution network is normal.

\section{Conclusion}

This paper introduces the commonly used evaluation methods as well as the fuzzy comprehensive evaluation method, and the corresponding theory and method of application. We use this method to evaluate the regional distribution network in the power supply bureau of a certain district, and get the corresponding score and state level. What's more, the final result is consistent with the actual. It not only shows the feasibility of these evaluation methods, but also provides the basis for the decision making.

\section{References}

[1] Tao Shen. Research on condition assessment method of power transformer[D]. Beijing: North China Electric Power University, 2007.In Chinese.

[2] Sanna Poyhonen, Marian Negrea. Antero Arkkio, Heikki. Fault diag-nostics of an electrical machine with multiple support vector classifiers. 2002. Proceedings of the 2002 IEEE International Symposium on In-telligent Control, Vancouver, Canada. 27-30 Oct. 2002 Pages:373 378.

[3] Bin Hua. A Dissertation Submitted in Partial Fulfillment of the Requirements[D].Wuhan: Huazhong University of science and technology, 2004.In Chinese.

[4] Chen-Fu Chien, Shi-Lin Chen, Yih-Shin Lin. Using Bayesian network for fault location on distribution feeder. IEEE Transactions on Power Delivery ， 2002,17(3):785 793

[5] Qian Wang. Study of the Comprehensive Assessment Method for The Power Transformer Condition in service with Fuzzy theory[D].Chongqing: Chongqing University, 2005.In Chinese.

[6] Ming Ding, Jing Zhang, Li Sheng-hu. A sequential monte-carlo simulation based reliability evaluation model for distribution network [J]. Power System Technology, 2004,28(3):38-42.In Chinese. 
[7] GoelL. Monte Carlosimulation basedre liability studies of adistribution testSysrem [J].EleetriePowersystemsreseareh,2000,54(1):55-65.

[8] Teng Zhang, Bo Zhang. Reliability evaluation of complex distribution system[J]. RELAY,2004,32(15):19一21.In Chinese.

[9] [10]A11anRN,Billinton R,Sjariefl,etal. Are Iiability test system for educational PurPose-basie distribution systemdata and results[J].IEEE Trans on Power Systems, 1991,6(2):813-830.

[10]BillintonR,Jonnavirhula5.AtestsystemforteachingoverallpowerSystemreliabilityassessmenr[J].I EEETransonPowerSystems,1996,11(4):1670-1676.

[11] Yan Zhang, Zhangchao Chen. Evaluation of a 35/10kV Electric Distribution System Reliability level[J].Journal of shanghai jiaotong university, 1995,29(5):198-201.In Chinese.

[12]Chaohong Bie, Xifan Wang .Reliability Analysis of Distribution Networks[J]China Power, 1997,30(5):10-13.In Chinese.

[13] Mingdong Li, Chaohong Bie, Xifan Wang. Study on reliability method of practical distribution network[J]. Northwest Electric Power Technology, 1992,1(2):1-7.In Chinese. 